\title{
Environmental fluctuation governs selection for plasticity in biofilm production
}

\author{
Jing Yan ${ }^{1,2,5}$, Carey D Nadell ${ }^{1,3,5}$ and Bonnie L Bassler ${ }^{1,4}$ \\ ${ }^{1}$ Department of Molecular Biology, Princeton University, Princeton, NJ, USA; ${ }^{2}$ Department of Mechanical and \\ Aerospace Engineering, Princeton University, Princeton, NJ, USA; ${ }^{3}$ Max Planck Institute for Terrestrial \\ Microbiology, Marburg, Germany and ${ }^{4}$ Howard Hughes Medical Institute, Chevy Chase, MD, USA
}

\begin{abstract}
Bacteria can grow in a free-swimming state, as planktonic cells, or in surface-attached communities, termed biofilms. The planktonic and biofilm growth modes differ dramatically with respect to spatial constraints, nutrient access, population density and cell-cell interactions. Fitness trade-offs underlie how successfully bacteria compete in each of these environments. Accordingly, some bacteria have evolved to be specialists in biofilm formation, while others specialize in planktonic growth. There are species, however, that possess flexible strategies: they can transition between the molecular programs required for biofilm formation and for planktonic growth. Such flexible strategies often sacrifice competitive ability against specialists in a given habitat. There is little exploration of the ecological conditions favoring the evolution of the flexible biofilm production strategy for bacteria in competition with specialist biofilm producers or specialist non-producers. Here, we study the human pathogen Vibrio cholerae, a flexible biofilm-former, as well as constitutive biofilm-producing and nonproducing mutants. We assess the fitness of these strains under biofilm conditions, planktonic conditions and conditions that demand the ability to transition between the two growth modes. We show that, relative to the specialists, the wild type is superior at dispersal from biofilms to the planktonic phase; however, this capability comes at the expense of reduced competitive fitness against constitutive biofilm producers on surfaces. Wild-type $V$. cholerae can outcompete the constitutive biofilm producers and non-producers if habitat turnover is sufficiently frequent. Thus, selection for phenotypic flexibility in biofilm production depends on the frequency of environmental fluctuations encountered by bacteria.

The ISME Journal (2017) 11, 1569-1577; doi:10.1038/ismej.2017.33; published online 24 March 2017
\end{abstract}

\section{Introduction}

Bacteria frequently live in surface-attached communities, called biofilms, which are embedded in a secreted polymer matrix (Hall-Stoodley et al., 2004; Nadell et al., 2009; Flemming and Wingender, 2010). The biofilm mode of bacterial growth is integral to the natural ecology of bacteria, and is additionally important in industry and medicine (Hall-Stoodley et al., 2004). Within the biofilm environment, cells are often immobilized, obtain nutrients passively by diffusion, and interact intimately with nearneighbors (Stewart and Franklin, 2008; Stewart, 2012; Nadell et al., 2016). The biofilm and planktonic niches differ fundamentally. In the latter, cells are often motile, actively seek resources via chemotaxis, and occur at distances far from neighboring cells (Stocker et al., 2008; Stocker and Seymour,

Correspondence: BL Bassler, Department of Molecular Biology, Princeton University, 329 Lewis Thomas Laboratory, Washington Road, Princeton, NJ 08544, USA.

E-mail: bbassler@princeton.edu

${ }^{5}$ These authors contributed equally to this work.

Received 9 November 2016; revised 29 December 2016; accepted 22 January 2017; published online 24 March 2017
2012). Shifts in the expression of up to $10 \%$ of the genome are common when bacteria transition between the planktonic and biofilm modes of growth (Whiteley et al., 2001; Beenken et al., 2004; Mikkelsen et al., 2007). In the case of biofilms, a substantial energetic investment is often required to produce the extracellular matrix (Nadell and Bassler, 2011). In the case of planktonic cells, assembling and operating flagella and mounting the chemosensory apparatus are metabolically expensive (MartínezGarcía et al., 2014). Thus, fitness trade-offs are incurred for each lifestyle, such that phenotypes conferring competitive success in biofilm environments carry selective disadvantages in the planktonic phase, and vice versa (Nadell and Bassler, 2011; Madsen et al., 2015; Oliveira et al., 2015).

Given that determinants of fitness differ in biofilm versus planktonic environments, one might expect bacteria to specialize in order to thrive in one environment or the other. Indeed, some bacterial species produce biofilms but are never actively motile, while other species appear incapable of making biofilms (Yawata et al., 2014). However, many bacteria-including common model organisms -are capable of switching between the biofilm and 
planktonic modes of growth. As opposed to their niche-specialist counterparts, such strains have plastic strategies (Snell-Rood, 2013): they invest in the regulatory machinery required to alternate between the different lifestyles depending on the circumstances. This remarkable flexibility in biofilm formation is well known, and, in the literature, is often assumed to be required for bacteria to successfully endure environmental variation. However, this assumption has not been explicitly tested experimentally, and, moreover, the minimal conditions that selectively favor flexible biofilm-production strategies when bacteria compete with other bacteria that possess specialist strategies have not been explored. Here we provide the first direct examination of the conditions favoring flexible versus fixed biofilm production.

Isolates of Vibrio cholerae, the etiologic agent of the human disease cholera, show broad variation in their propensities to make biofilms (Yawata et al., 2014; Chowdhury et al., 2016): some strains are constitutive biofilm-producers, some strains make little to no biofilms, while other strains including well-studied model strains are flexible strategists. With respect to $V$. cholerae strains that are flexible in their biofilm-forming capabilities, planktonic growth and biofilm formation are both crucial for successful proliferation within and outside of human hosts (Nelson et al., 2009; Fong et al., 2010; Utada et al., 2014; Teschler et al., 2015). There is little underlying understanding for the wide variation in biofilm production strategies observed in $V$. cholerae strains in particular, or across different bacterial species more broadly. Here, we examine a commonly studied wild isolate of $V$. cholerae that is capable of flexible biofilm production, as well as two derived strains, one that produces biofilm matrix constitutively and one that is incapable of biofilm matrix production. Using a variety of competition experiments, we define how these different strategies confer evolutionary fitness, and we identify the environmental conditions under which flexible biofilm production is favored by selection.

\section{Materials and methods}

Strains and media

All $V$. cholerae strains used in this study are derivatives of the wild-type Vibrio cholerae $\mathrm{O} 1$ biovar El Tor strain C6706. Additional mutations were engineered into this $V$. cholerae strain using Escherichia coli S17- $\lambda$ pir carrying pKAS32 or its derivatives (Skorupski and Taylor, 1996). All strains were grown in lysogeny broth (LB) medium at $37^{\circ} \mathrm{C}$ with shaking overnight. Unless otherwise indicated, biofilm experiments were performed in M9 minimal medium, supplemented with $0.5 \%$ casamino acids, $2 \mathrm{mM} \mathrm{MgSO}_{4}$, and $100 \mu \mathrm{M} \mathrm{CaCl}$. All media contain $6 \mu \mathrm{gl}^{-1}$ polymyxin B. Although V. cholerae C6706 is resistant to polymyxin $\mathrm{B}$, we found that low doses, similar to other antibiotics (Hoffman et al., 2005), encourage biofilm growth. A detailed strain list is provided in Supplementary Table 1.

\section{Statistics}

Four biological replicates were performed for each experiment unless indicated otherwise; error bars correspond to standard errors throughout the text. For biomass quantification, at least 6 different locations (technical replicates) with $x y$ dimensions of $250 \mu \mathrm{m} \times 250 \mu \mathrm{m}$ were sampled and averaged for each biological replicate. For cell counting, after placing liquid samples on microscope slides, 10 different locations with $x y$ dimensions of $250 \mu \mathrm{m} \times 250 \mu \mathrm{m}$ were sampled for each biological replicate. All statistical analyses are unpaired $t$-tests with Welch's correction.

\section{Biofilm growth procedure for isolated strains}

$V$. cholerae strains were grown overnight at $37^{\circ} \mathrm{C}$ in liquid LB medium with shaking, back-diluted 30 -fold and grown for an additional $2 \mathrm{~h}$ with shaking in M9 medium until early exponential phase $\left(O D_{600}=0.2-0.4\right)$. The $\mathrm{OD}_{600}$ of each culture was measured and the culture was back-diluted into fresh M9 medium to a final $\mathrm{OD}_{600}$ of 0.033 . The inoculum was then transferred into planar microfluidic devices measuring $1 \mathrm{~cm}$ in length, $400 \mu \mathrm{m}$ in width and $60 \mu \mathrm{m}$ in height. After $2 \mathrm{~h}$ of incubation, fresh medium was provided continuously with a syringe pump at a flow rate of $0.6 \mu \mathrm{min}^{-1}$ (corresponding to an average flow velocity of $417 \mu \mathrm{m} \mathrm{s}^{-1}$ inside the chamber). The biofilms were grown at ambient room temperature $\left(25-30^{\circ} \mathrm{C}\right)$. To measure biofilm population sizes, the biofilms were imaged by confocal microscopy at $z$-intervals of $3 \mu \mathrm{m}$, to ensure minimal overlap between consecutive optical sections. The images obtained in this manner were thresholded and converted to cell counts per unit area using protocols developed previously (Drescher et al., 2014; Nadell et al., 2015). In brief, bacterial biomass was distinguished from matrix material and background fluorescence. Each cross-sectional image was thresholded and converted to cell counts based on the average size of a bacterial cell, which was measured separately in a monolayer sample. Biofilm growth involving dispersal mutants was performed in medium containing $10 \mu \mathrm{M} \mathrm{CaCl} \mathrm{Cl}_{2}$ instead of $100 \mu \mathrm{M}$ to promote increased VPS-dependent biofilm growth (Bilecen and Yildiz, 2009).

\section{Procedure for dispersal assay}

After $12 \mathrm{~h}$ of growth, biofilms were imaged for population size quantification. Immediately after imaging, the outlet tubing of the microfluidic channel was cut to a minimal length, and the flow was interrupted for $2 \mathrm{~h}$. A fast flow pulse $\left(5 \mu \mathrm{min}^{-1}\right.$, corresponding to a flow velocity of $\sim 3.5 \mathrm{~mm} \mathrm{~s}^{-1}$ ) was 
applied briefly ( $2 \mathrm{~min}$; which was sufficient for the effluent to become optically clear) to flush out all cells that had dispersed during the nutrient limitation period. The biofilms remaining in the microfluidic channel were imaged again at the original locations. The liquid drop exiting the effluent tubing was collected, diluted in series into M9 medium (without nutrients), plated onto LB agar and allowed to grow overnight at $30^{\circ} \mathrm{C}$. Colony-forming units were counted manually and used to calculate the total cell number in the original effluents. The biomass quantification procedure described above was used to assess the biomass prior to and after nutrient limitation.

\begin{abstract}
Microscopy
Microscopy was performed with a Borealis confocal system with a Yokogawa CSU-X1 confocal spinning disk unit (Tokyo, Japan) mounted on a Nikon Ti-E inverted microscope (Tokyo, Japan). Images were acquired with a $60 \times$ water objective with a numerical aperture of 1.2 and captured with an Andor iXon 897 EMCCD camera. Three diode lasers (445 nm, $543 \mathrm{~nm}$ and $592 \mathrm{~nm}$ ) were used sequentially along with customized dichroic filters (Chroma, Marlborough, MA, USA) for detection, providing good spectral separation for the three fluorescent proteins used in the study. To image fine structures within individual biofilm clusters, an additional $\times 1.5$ magnification lens was placed between the microscope and the spinning disk, and the $z$-step size was adjusted to $0.2 \mu \mathrm{m}$. All experimental images presented in this work are raw images from this step, rendered by Nikon Elements software.
\end{abstract}

\section{Biofilm and dispersal competition assay}

We competed the WT $V$. cholerae strain carrying constitutively expressed $m T F P 1$, the extracellular polymeric substances $\left(\mathrm{EPS}^{+}\right)$strain with constitutively expressed mKate 2 and the $\mathrm{EPS}^{-}$strain with constitutively expressed $m K O$, all of which were inserted into the chromosomal lacZ locus. Previous work has established that the production of these fluorescent proteins does not affect the growth rate of $V$. cholerae (Nadell and Bassler, 2011; Drescher et al., 2014; Nadell et al., 2015). Overnight cultures were first grown in the presence of glass beads (4 mm, MP Biomedicals, Santa Ana, CA, USA) and vigorously shaken, back-diluted 30-fold and grown with shaking in M9 medium with glass beads to $\mathrm{OD}_{600}=0.2-0.4$. The regrown cultures were transferred to Eppendorf tubes with smaller glass beads (acid-washed, 425-500 $\mu \mathrm{m}$, Sigma, St Louis, MO, USA), and vigorously bead-bashed on a vortex mixer. These steps were necessary to disassemble cell clusters that had formed in the liquid cultures, if any, for accurate $\mathrm{OD}_{600}$ measurements and $\mathrm{OD}_{600}$ equalization. An inoculum with a total $\mathrm{OD}_{600}$ of 0.1 , regardless of the composition, was prepared after the measurement. The inoculum was then transferred into planar microfluidic chambers and incubated for $2 \mathrm{~h}$, after which fresh medium was provided continuously with a syringe pump. For biofilm competitions, images were taken of biofilms in the microfluidic chambers after $16 \mathrm{~h}$ of growth. The image-processing-based biomass quantification procedure described above was used separately for each strain in the co-cultures, and subsequently, to define each strain's population frequency. In the case of dispersal competitions, after $12 \mathrm{~h}$ of biofilm growth, flow was interrupted for $2 \mathrm{~h}$, followed by a fast flow pulse to flush out all dispersed cells (see above). Effluents were collected, mixed with $80 \%$ glycerol and temporarily stored at $-80^{\circ} \mathrm{C}$. The frequency of each strain in the effluent was measured later by two methods: (1) cell counting via microscopy. In this case, $5 \mu \mathrm{l}$ of effluents were placed between coverslips and agar pads made from M9 medium without nutrient. (2) serial dilution and plating. In this case, a gel imager (Image Quant LAS 4000) with different filter sets was used to differentiate the strains on LB plates, and to count the number of colonies of each strain.

\section{Liquid growth curve and competition}

Overnight cultures of each strain grown in LB were back-diluted 30-fold into M9 medium, and regrown for $2 \mathrm{~h}$. The regrown cultures were back-diluted again into $15 \mathrm{ml}$ M9 medium with glass beads in a $50 \mathrm{ml}$ centrifuge tube to yield an initial $\mathrm{OD}_{600}$ of 0.0004 . The cultures were then grown on a shaker at $30^{\circ} \mathrm{C}$, and were additionally manually shaken and mixed by vortex every hour. Every $2 \mathrm{~h}, \mathrm{OD}_{600}$ was measured. For competitions in liquid, cultures were prepared as described above for biofilm competition experiments, to a total $\mathrm{OD}_{600}$ of 0.3 per inoculating mixture. $10 \mu \mathrm{l}$ of the inoculum was added to $500 \mu \mathrm{l}$ of fresh M9 medium with small glass beads in a $1.5 \mathrm{ml}$ Eppendorf tube, which was subsequently rotated end over end at $30^{\circ} \mathrm{C}$. Every $4 \mathrm{~h}$, the culture was mixed by vortex. After $8 \mathrm{~h}$ of liquid growth, $10 \mu \mathrm{l}$ of this culture was back-diluted into $500 \mu \mathrm{l}$ of fresh M9 medium with small glass beads. After $16 \mathrm{~h}$ of growth in total, the liquid culture was temporarily frozen after addition of $80 \%$ of glycerol. The strain frequencies in the culture were later analyzed by imaging samples of the culture by confocal microscopy.

\section{Experiments simulating a fluctuating environment} The biofilm growth procedure was performed as described above, with an initial inoculum containing a 1:1:1 mixture of WT, EPS ${ }^{+}$and $\mathrm{EPS}^{-}$strains at a total $\mathrm{OD}_{600}$ of 0.1 . For the zero-disturbance experimental treatment, biofilm samples were imaged at 34-36 h. For the one-disturbance experimental treatment, biofilms were imaged after $12 \mathrm{~h}$ of biofilm growth. The imaging duration was $2 \mathrm{~h}$, after which 
flow was interrupted. The outlet tubing was removed, and new tubing was used to connect the outlet of the initial chamber to a new empty microfluidic chamber. After $2 \mathrm{~h}$ of nutrient limitation, flow was resumed to pump the dispersed cells from the initial chamber to the new chamber. The new chamber was incubated for $2 \mathrm{~h}$, and then fresh medium was provided. After another $12 \mathrm{~h}$ of biofilm growth, biomasses were again imaged. For the three-disturbance experimental treatment, the identical procedure was performed as described above for the one-disturbance treatment, except all the time scales were halved and the disturbance and chamber recolonization procedure was repeated 3 times instead of once. The total durations of the experiments for all three-disturbance conditions are roughly identical (35-36 h).

\section{Results}

Biofilm production, planktonic growth and dispersal To explore the fitness consequences of constitutive versus flexible biofilm production strategies, we measured the biofilm and planktonic growth properties of three $V$. cholerae strains: the wild type (designated WT), which is able to transition between biofilm and planktonic modes of growth (McDougald et al., 2012; Teschler et al., 2015); a strain that constitutively produces extracellular matrix due to a point mutation $\left(v p v C^{W 240 R}\right)$ that results in elevated levels of the second messenger molecule c-di-GMP and, in turn, hyper-secretion of the biofilm matrix (designated EPS ${ }^{+}$; Beyhan and Yildiz, 2007); and a strain that is unable to produce extracellular matrix due to deletion of the vpsL gene, required for synthesis of a key matrix polysaccharide (designated EPS'; Fong et al., 2010). When grown in isolation, the $\mathrm{EPS}^{+}$strain is the strongest biofilm-producer, accumulating threefold more biomass in microfluidic channels than the WT and 10-fold more biomass than the EPS- ${ }^{-}$strain, which only forms a loosely attached monolayer (Figure 1a).

In planktonic cultures, which were periodically shaken with beads to ensure that cells did not grow in clusters (Nadell and Bassler, 2011), the WT and the EPS $^{-}$strain grew at indistinguishable rates that were $\sim 20 \%$ higher than the growth rate of the EPS ${ }^{+}$ strain (Figure 1b). In liquid, the WT strain represses biofilm-associated genes (Beyhan et al., 2007; Papenfort et al., 2015), and hence, grows at the same rate as the EPS ${ }^{-}$strain. Importantly, the growth rate differences between our three strains could be attributed to their differences in matrix secretion, rather than differences in c-di-GMP levels. Specifically, deletion of the vpsL gene in the strain with elevated c-di-GMP level restored the growth rate to that of the WT in liquid medium (Supplementary Figure 1). These results are consistent with our previous report documenting that extracellular matrix production enhances fitness on surfaces but at a cost to maximum growth rate in liquid (Nadell and Bassler, 2011). In sum, WT cells are inferior to
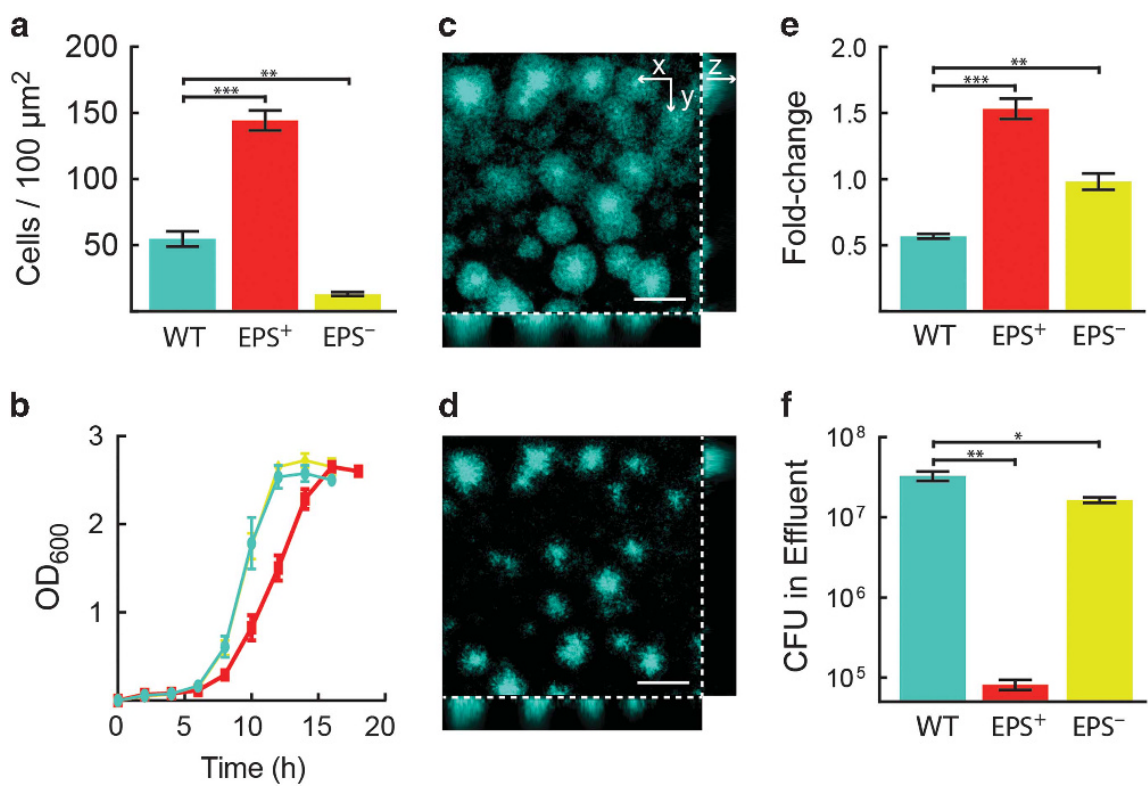

Figure 1 Growth characteristics of WT, EPS ${ }^{+}$and EPS - $V$. cholerae strains. In all panels, WT is teal, EPS ${ }^{+}$is red and EPS- is yellow. $n=4$ biological replicates for all experiments. (a) Number of biofilm cells per unit area of all strains after $16 \mathrm{~h}$ growth under biofilm-forming conditions. (b) Growth curves in shaken liquid culture. (c and d) Representative confocal images of biofilms formed by WT V. cholerae cells constitutively expressing mTFP1, before (c) and after (d) nutrient limitation for $2 \mathrm{~h}$. The cross-sectional $x y$-view at $3 \mu \mathrm{m}$ above the surface is shown in the upper left large panel and two orthogonal z-projections are shown on the bottom and right. Scale bar: $50 \mu m$. (e) Fold-change in biofilm cell counts after $2 \mathrm{~h}$ nutrient limitation. (f) Colony-forming units (CFU) in the effluents collected immediately after the nutrient limitation period. Data are represented as mean \pm s.e.m. All statistical comparisons are unpaired $t$-tests. ${ }^{* * *}$ denotes $P<0.0005$, * denotes $P<0.005$, and * denotes $P<0.05$. 
EPS $^{+}$cells under biofilm-forming conditions, and they do not have an advantage over EPS ${ }^{-}$cells in planktonic growth.

Previous work has suggested that WT $V$. cholerae biofilm-dwelling cells are primed for dispersal when they become nutrient-limited (for example, when fluid flow over biofilms is halted; Nielsen et al., 2006; Müller et al., 2007). We measured how differences in EPS production among our three strains influence dispersal using two assays (Figures 1c-f). Biofilms of each strain were grown in isolation, after which (1) their biomasses were measured before and after nutrient limitation in the microfluidic channels, and (2) the total numbers of dispersed cells were measured in the effluent downstream of each biofilm immediately following nutrient limitation. The WT biofilm biomass declined by $\sim 50 \%$ following nutrient limitation (Figure 1e): the WT biofilm clusters shrank in size and they appeared to lose cells from both the outer periphery and from the interior (Figures 1c and d). EPS $^{+}$ biofilms, by contrast, continued to grow before nutrient was completely depleted, yielding a $~ 50 \%$ increase in biomass by the conclusion of the experiment (Figure 1e). EPS ${ }^{-}$cells produced monolayer biofilms that did not significantly change in size after nutrient limitation (Figure 1e). The effluents from the nutrient-limited biofilms contained 2fold and 400-fold more WT cells than EPS ${ }^{-}$and EPS ${ }^{+}$ cells, respectively (Figure 1f). These data indicate that the EPS ${ }^{-}$strain produces monolayer biofilms from which newly divided daughter cells detach continuously, whereas $\mathrm{EPS}^{+}$cells are severely impaired for dispersal due to over-production of the matrix (Nadell and Bassler, 2011). The WT strain, by contrast, produces sufficient matrix to accumulate 3D biofilms while also effectively dispersing following nutrient limitation. WT dispersal depended on secretion of the extracellular enzyme RbmB, which has previously been implicated in EPS digestion (Fong and Yildiz, 2007), as well as extracellular proteases including HapA and IvaP that target the protein components of the $V$. cholerae biofilm matrix (Supplementary Figures 2 and 3; Smith et al., 2015).

Pairwise and three-way competition between WT, EPS and EPS $^{-}$strains

The above results establish that WT, $\mathrm{EPS}^{+}$and EPS ${ }^{-}$ strains of $V$. cholerae differ in their relative abilities to grow in the planktonic phase and to form and disperse from biofilms when in mono-culture. We investigated the consequences of these differences using pairwise and three-way competitions in the different environments, that is, in continuous liquid culture, continuous biofilm culture and in the biofilm dispersal assay. We varied the initial strain ratios systematically and measured the final population composition after $16 \mathrm{~h}$ (Supplementary Data 1). The data were used to construct phase plane diagrams, from which one can infer the stable population steady state in each environmental condition.

When grown in shaken liquid co-culture, WT and EPS $^{-}$cells remain at the same ratio at which they were inoculated (Supplementary Figure 4). The right edge in Figure 2a defining all two-strain ratios of the WT and the EPS ${ }^{-}$strain is thus a line of neutrally stable equilibria. However, WT and EPS ${ }^{-}$cells are both competitively superior to the constitutively matrix-producing $\mathrm{EPS}^{+}$strain in shaken liquid (Figure 2a and Supplementary Figure 5). Specifically, in liquid cultures containing mixtures of all three strains, WT and EPS ${ }^{-}$strains outcompete the $\mathrm{EPS}^{+}$strain, such that the relative ratio of WT to EPS changes minimally while the $\mathrm{EPS}^{+}$population fraction declines toward zero (Figure 2a). This result is consistent with our above growth rate measurements (Figure 1b). When grown in biofilm-forming conditions, by contrast, uniform positive selection occurs for the EPS ${ }^{+}$strain (Figure 2b and Supplementary Figure 5), that is, EPS ${ }^{+}$cells approach $100 \%$ of the biofilm population starting from any initial condition. Notably, although the WT produces matrix and forms biofilms, it is outcompeted by the EPS ${ }^{+}$strain in biofilm co-culture (Supplementary Figure 6). The WT strain, in turn, outcompetes the EPS ${ }^{-}$strain in co-inoculated biofilms (Figure 2b and Supplementary Figure 6). Thus, each strain's fitness in the biofilm-forming environment tracks with its level of extracellular matrix production relative to its competitors (Xavier and Foster, 2007; Nadell et al., 2008). Indeed, matrix-secreting strains of a variety of bacterial species spatially self-segregate from, laterally displace, and outcompete strains that produce less or no matrix (Xavier and Foster, 2007; Nadell and Bassler, 2011; Kim et al., 2014; Madsen et al., 2015; Nadell et al., 2015; Schluter et al., 2015; Drescher et al., 2016; Irie et al., 2016; Kragh et al., 2016; Yan et al., 2016).

To test the ability of the different strains to transition between biofilm and planktonic states in a competitive context, we assessed the composition of the effluent immediately following nutrient limitation of biofilms co-inoculated with different ratios of the three strains. The WT clearly dominates in direct competition with $\mathrm{EPS}^{+}$and $\mathrm{EPS}^{-}$cells (Figure 2c, Supplementary Figures 5 and 7). Presumably, the WT outcompetes the other strains because it can produce sufficient matrix to form a 3D biofilm structure during the growth period, and subsequently it can digest matrix components and disperse a large fraction of its biofilm biomass into the liquid phase during the nutrient limitation period. Consistent with this interpretation, WT cell clusters shrink during the nutrient limitation, whereas the $\mathrm{EPS}^{+}$cell clusters do not (Figures 2d and e). Overall, our experiments suggest that the WT is inferior in the biofilm phase when in competition with stronger matrix-producers, but the WT is superior in its ability to transition between the biofilm and planktonic growth modes. 


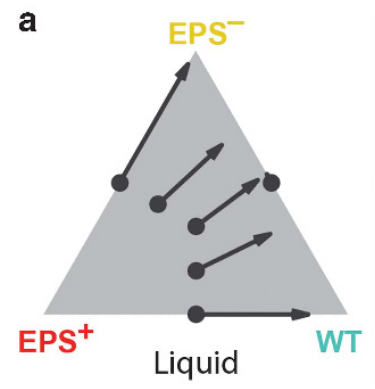

d

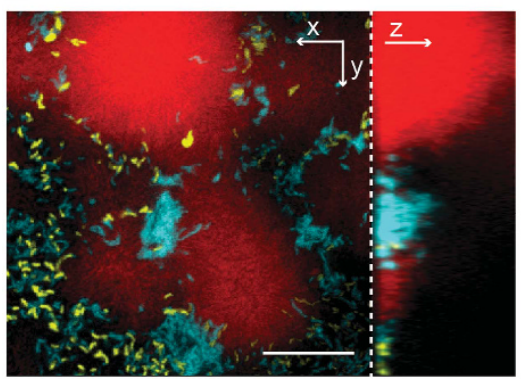

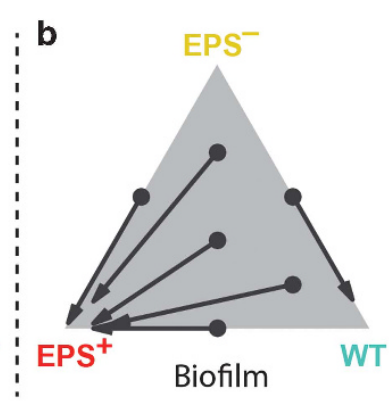

e

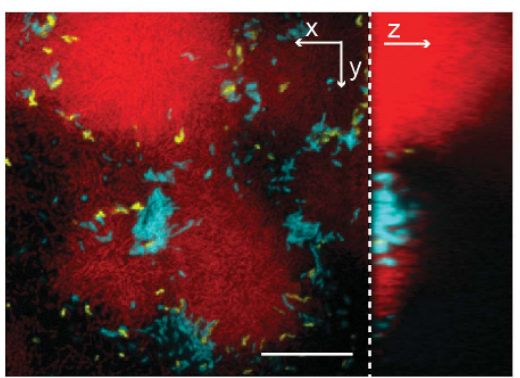

Figure 2 Competition of $V$. cholerae strains with different biofilm production strategies. In all panels, teal, red and yellow correspond to WT, EPS ${ }^{+}$and EPS ${ }^{-}$cells, respectively. (a-c) Three-way competition between WT, EPS ${ }^{+}$and EPS $^{-}$strains in shaken liquid culture (a), under biofilm-forming conditions (b) and under biofilm dispersal conditions (c). The composition of each initial inoculum is represented by the black dots on the ternary plots. The average final compositions were measured and are denoted by the end points of each arrow. The full data are presented in Supplementary Figure 5. (d and e) Representative $12 \mathrm{~h}$ biofilm structures before (d) and after (e) $2 \mathrm{~h}$ of nutrient

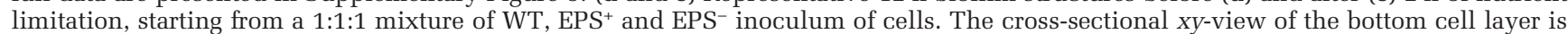
shown on the left and the $y z$ side view is shown on the right. Scale bar: $20 \mu \mathrm{m}$. Note the decrease in size of the WT biofilms from $\mathbf{d}$ to e.

\section{A flexible biofilm formation-dispersal strategy is favored in fluctuating environments}

The above experiments examine competition under selection in a single habitat. The natural environment in which $V$. cholerae resides is subject to temporal variation in habitat type and quality, as well as sudden influxes or depletions of resources (Teschler et al., 2015; Conner et al., 2016). We explored the consequences of differential matrix production and dispersal abilities in alternating environments. To do this, we grew 1:1:1 mixtures of the WT, EPS ${ }^{+}$and EPS ${ }^{-}$strains under biofilmforming conditions for a fixed total amount of time, and we varied the number of disturbance and biofilm re-colonization events between treatments (either 0 , 1 or 3). For each disturbance event, fluid flow was interrupted to deplete nutrients from the biofilm cells prior to pulsing the biofilm effluent into another microfluidic chamber. The goal was to simulate the natural transition of $V$. cholerae from an existing biofilm with diminishing resources to a new region rich with resources with an intervening planktonic phase. Biofilms were imaged immediately prior to each disturbance event, and again at the end of the experiment (Figure 3).

When there were no disturbance events over $36 \mathrm{~h}$, the $\mathrm{EPS}^{+}$strain approached $97 \%$ of the population (Figure 3a, left). With the introduction of onedisturbance event, the population fraction of the $\mathrm{EPS}^{+}$strain declined to $93 \%$, the WT strain rose to a detectable minority at $7 \%$ and the EPS $^{-}$strain remained nearly undetectable (Figure 3a, middle).
When three-disturbance events occurred over $36 \mathrm{~h}$, the WT comprised $75 \%$ of the population and was increasing at the conclusion of the experiment (Figure 3a, right). We expect WT cells to approach fixation in experiments with longer duration or higher disturbance rate, but these could not be performed at present due to technical constraints.

Figure $3 \mathrm{~b}$ illustrates the time-resolved frequencies of WT, EPS ${ }^{+}$and EPS ${ }^{-}$at 4 time points during the experimental condition of three-disturbance/re-colonization events over $36 \mathrm{~h}$. WT increased in frequency at every sampling point. The frequency of $\mathrm{EPS}^{+}$cells trended downward overall, but oscillated from onedisturbance event to another, which appeared to be due to fluctuations in the initial population density after re-colonization of new biofilm chambers. The EPS $^{-}$strain increased in frequency initially, but over the course of the experiment, declined to a minority fraction of $14 \%$ of the population. We presume this decrease is due to the fact that the EPS- strain is outcompeted by WT and $\mathrm{EPS}^{+}$cells during biofilm growth, ultimately reducing its ability to seed new chambers following disturbance.

These results show that the WT capability to form and disperse from biofilms is selectively favored under high variation and turnover in habitat type over time, which is consistent with environmental conditions known to be important for $V$. cholerae in its natural state (Conner et al., 2016). In the environment, $V$. cholerae resides on and consumes chitin particles with finite lifetimes (Meibom et al., 2004; Stocker and Seymour, 2012; Drescher et al., 
a

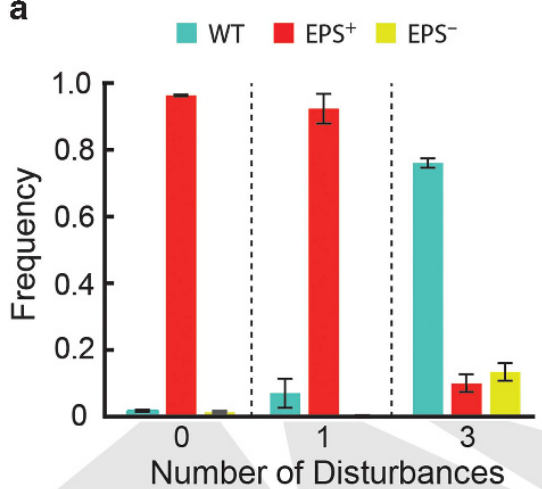

b Biofilm Imaging Disturbance

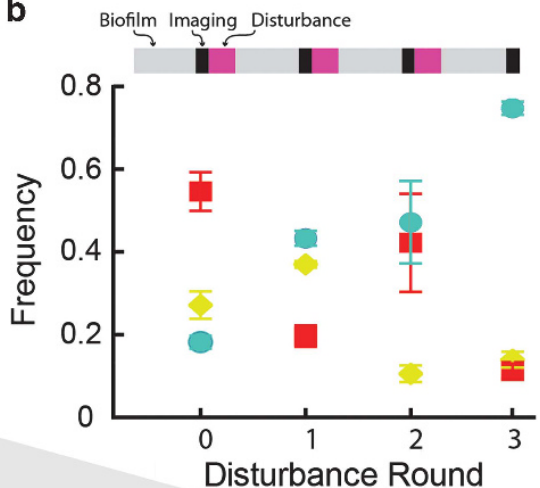

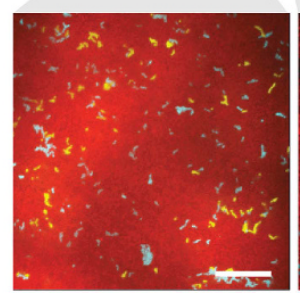
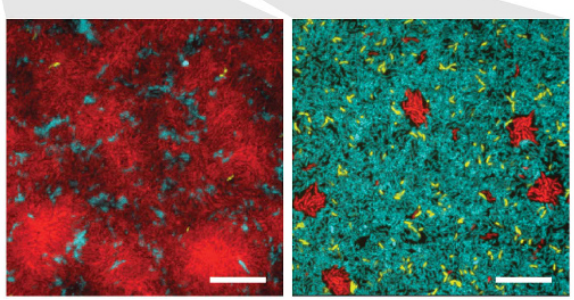

Figure 3 A fluctuating environment selects for WT V. cholerae with a plastic biofilm production strategy. Cultures were initiated with a 1:1:1 mixture of WT:EPS ${ }^{+}:$EPS $^{-}$cells in microfluidic devices. Chambers were subjected to a period of nutrient limitation (1/6 of the biofilm growth time) after which, cells were allowed to disperse to a new chamber. In all panels, WT is teal, EPS ${ }^{+}$is red and EPS ${ }^{-}$is yellow. (a) Final strain composition of cultures in competition experiments with different disturbance frequencies. Below: representative biofilm images taken of the bottom cell layer after competition with continuous nutrient provision (left), with disturbance events every $12 \mathrm{~h}$ (middle) and with disturbance events every $6 \mathrm{~h}$ (right). Images are single optical sections through the basal layers of the biofilms. Scale bar: $20 \mu \mathrm{m}$. (b) Frequency of the WT, EPS ${ }^{+}$and EPS ${ }^{-}$strains during competition with disturbance events every $6 \mathrm{~h}$. The timeline of the experiment is displayed in the bar above the data: light gray corresponds to biofilm-forming conditions in a microfluidic device; black refers to a sampling period when biofilms were imaged, and magenta corresponds to a disturbance event in which cells were depleted for nutrients for one hour. Following that, biofilm effluents were used to colonize new chambers for $1 \mathrm{~h}$, followed by another phase of biofilm growth. WT: teal circles; EPS ${ }^{+}$red squares; and EPS ${ }^{-}$cells: yellow diamonds. Data are represented as mean \pm s.e.m $(n=3-4)$.

2014). During infection, $V$. cholerae establishes biofilms in the host intestine and then disperses after causing the lumen to become flooded with fluid (Fong et al., 2010; Millet et al., 2014; Teschler et al., 2015; Sengupta et al., 2016). Both of these natural environments involve repeated transitions between the planktonic and biofilm growth modes. Our experiments show that the plastic biofilm production strategy of WT $V$. cholerae is particularly well suited to such fluctuating environments.

\section{Discussion}

Understanding the conditions that select for phenotypic flexibility and diverse habitat occupation, as opposed to fixed specialization in one particular habitat, is of long-standing interest in ecology and evolution (Wilson and Yoshimura, 1994; Kassen, 2002; Egas et al., 2004). A major factor that limits the evolution of generalist behaviors is the compromised ability to compete against specialists in any particular environment (Devictor et al., 2008; Ali and Agrawal, 2012; Lowe and McPeek, 2014). Here, we demonstrate that WT $V$. cholerae transitions effectively from the biofilm to the planktonic state using matrix-degrading enzymes, but this capability comes at the expense of its competitive ability against constitutive biofilm producers on surfaces. This compromise is offset when disturbance events are frequent, which allows WT $V$. cholerae cells to become the majority and, we expect, competitively eliminate constitutive biofilm producers and nonproducers over the long run. Our results suggest that the temporal stability of environments in which particular $V$. cholerae isolates reside is key to determining whether or not biofilm regulation will be favored by natural selection. It is important to note that strains of $V$. cholerae and other Vibrio spp. that regulate matrix production as well as strains that make minimal biofilms or that constitutively secrete matrix have been isolated in nature (Yawata et al., 2014; Chowdhury et al., 2016). We predict that an important determinant underlying this variation in matrix production strategy is the lifetime of resource patches (Nadell et al., 2008), with longevity favoring constitutive phenotypes and rapid turnover favoring flexible matrix production.

\section{Conflict of Interest}

The authors declare no conflict of interest.

\section{Acknowledgements}

This work was supported by the Alexander von Humboldt Foundation (CDN), Howard Hughes Medical Institute (BLB), NIH Grant 2R37GM065859 (BLB) and National 
Science Foundation Grant MCB-0948112 (BLB). JY holds a Career Award at the Scientific Interface from the Burroughs Wellcome Fund.

\section{Author contributions}

CDN and JY initiated this project. JY and CDN constructed the strains. JY performed the experiments. JY, CDN and BLB analyzed the data. CDN, JY and BLB wrote the manuscript.

\section{References}

Ali JG, Agrawal AA. (2012). Specialist versus generalist insect herbivores and plant defense. Trends Plant Sci 17: 293-302.

Beenken KE, Dunman PM, McAleese F, Macapagal D, Murphy E, Projan SJ et al. (2004). Global gene expression in Staphylococcus aureus biofilms. J Bacteriol 186: 4665-4684.

Beyhan S, Bilecen K, Salama SR, Casper-Lindley C, Yildiz FH. (2007). Regulation of rugosity and biofilm formation in Vibrio cholerae: comparison of VpsT and VpsR regulons and epistasis analysis of vpsT, vpsR, and hapR. J Bacteriol 189: 388-402.

Beyhan S, Yildiz FH. (2007). Smooth to rugose phase variation in Vibrio cholerae can be mediated by a single nucleotide change that targets c-di-GMP signalling pathway. Mol Microbiol 63: 995-1007.

Bilecen K, Yildiz FH. (2009). Identification of a calciumcontrolled negative regulatory system affecting Vibrio cholerae biofilm formation. Environ Microbiol 11: 2015-2029.

Chowdhury G, Bhadra RK, Bag S, Pazhani GP, Das B, Basu P et al. (2016). Rugose atypical Vibrio cholerae O1 El Tor responsible for 2009 cholera outbreak in India. J Med Microbiol 65: 1130-1136.

Conner JG, Teschler JK, Jones CJ, Yildiz FH. (2016). Staying alive: Vibrio cholerae's cycle of environmental survival, transmission, and dissemination. Microbiol Spectr 4 doi:10.1128/microbiolspec.VMBF-0015-2015.

Devictor V, Julliard R, Jiguet F. (2008). Distribution of specialist and generalist species along spatial gradients of habitat disturbance and fragmentation. Oikos 117: 507-514.

Drescher K, Nadell CD, Stone HA, Wingreen NS, Bassler BL. (2014). Solutions to the public goods dilemma in bacterial biofilms. Curr Biol 24: 50-55.

Drescher K, Dunkel J, Nadell CD, van Teeffelen S, Grnja I, Wingreen NS et al. (2016). Architectural transitions in Vibrio cholerae biofilms at single-cell resolution. Proc Natl Acad Sci USA 113: E2066-E2072.

Egas M, Dieckmann U, Sabelis MW. (2004). Evolution restricts the coexistence of specialists and generalists: the role of trade-off structure. Am Nat 163: 518-531.

Flemming H-C, Wingender J. (2010). The biofilm matrix. Nat Rev Microbiol 8: 623-633.

Fong JCN, Yildiz FH. (2007). The rbmBCDEF gene cluster modulates development of rugose colony morphology and biofilm formation in Vibrio cholerae. J Bacteriol 189: $2319-2330$.
Fong JCN, Syed KA, Klose KE, Yildiz FH. (2010). Role of Vibrio polysaccharide (vps) genes in VPS production, biofilm formation and Vibrio cholerae pathogenesis. Microbiol 156: 2757-2769.

Hall-Stoodley L, Costerton JW, Stoodley P. (2004). Bacterial biofilms: from the natural environment to infectious diseases. Nat Rev Microbiol 2: 95-108.

Hoffman LR, D'Argenio DA, MacCoss MJ, Zhang Z, Jones RA, Miller SI. (2005). Aminoglycoside antibiotics induce bacterial biofilm formation. Nature 436: 1171-1175.

Irie Y, Roberts A, Kragh KN, Gordon VD, Hutchison J, Allen RJ et al. (2016). The Pseudomonas aeruginosa PSL polysaccharide is a social but non-cheatable trait in biofilms. bioRxiv: doi: https://doi.org/10.1101/049783.

Kassen R. (2002). The experimental evolution of specialists, generalists, and the maintenance of diversity. J Evol Biol 15: 173-190.

Kim W, Racimo F, Schluter J, Levy SB, Foster KR. (2014). Importance of positioning for microbial evolution. Proc Natl Acad Sci USA 111: E1639-E1647.

Kragh KN, Hutchison JB, Melaugh G, Rodesney C, Roberts AEL, Irie Y et al. (2016). Role of multicellular aggregates in biofilm formation. mBio 7: e00237.

Lowe WH, McPeek MA. (2014). Is dispersal neutral? Trends Ecol Evol 29: 444-450.

Madsen JS, Lin Y-C, Squyres GR, Price-Whelan A, de Santiago Torio A, Song A et al. (2015). Facultative control of matrix production optimizes competitive fitness in Pseudomonas aeruginosa PA14 biofilm models. Appl Environ Microbiol 81: 8414-8426.

Martínez-García E, Nikel PI, Chavarría M, Lorenzo V. (2014). The metabolic cost of flagellar motion in Pseudomonas putida KT2440. Environ Microbiol 16: 291-303.

McDougald D, Rice SA, Barraud N, Steinberg PD, Kjelleberg S. (2012). Should we stay or should we go: mechanisms and ecological consequences for biofilm dispersal. Nat Rev Micro 10: 39-50.

Meibom KL, Li XBB, Nielsen AT, Wu CY, Roseman S, Schoolnik GK. (2004). The Vibrio cholerae chitin utilization program. Proc Natl Acad Sci USA 101: 2524-2529.

Mikkelsen H, Duck Z, Lilley KS, Welch M. (2007). Interrelationships between colonies, biofilms, and planktonic cells of Pseudomonas aeruginosa. J Bacteriol 189: 2411-2416.

Millet YA, Alvarez D, Ringgaard S, von Andrian UH, Davis BM, Waldor MK. (2014). Insights into Vibrio cholerae intestinal colonization from monitoring fluorescently labeled bacteria. PLoS Pathog 10: e1004405.

Müller J, Miller MC, Nielsen AT, Schoolnik GK, Spormann AM. (2007). vpsA-and luxO-independent biofilms of Vibrio cholerae. FEMS Microbiol Lett 275: 199-206.

Nadell CD, Xavier JB, Levin SA, Foster KR. (2008). The evolution of quorum sensing in bacterial biofilms. PLoS Biol 6: e14.

Nadell CD, Xavier JB, Foster KR. (2009). The sociobiology of biofilms. FEMS Microbiol Rev 33: 206-224.

Nadell CD, Bassler BL. (2011). A fitness trade-off between local competition and dispersal in Vibrio cholerae biofilms. Proc Natl Acad Sci USA 108: 14181-14185.

Nadell CD, Drescher K, Wingreen NS, Bassler BL. (2015). Extracellular matrix structure governs invasion resistance in bacterial biofilms. ISME J 9: 1700-1709. 
Nadell CD, Drescher K, Foster KR. (2016). Spatial structure, cooperation, and competition in bacterial biofilms. Nat Rev Microbiol 14: 589-600.

Nelson EJ, Harris JB, Glenn Morris J, Calderwood SB, Camilli A. (2009). Cholera transmission: the host, pathogen and bacteriophage dynamic. Nat Rev Microbiol 7: 693-702.

Nielsen AT, Dolganov NA, Otto G, Miller MC, Wu CY, Schoolnik GK. (2006). RpoS controls the Vibrio cholerae mucosal escape response. PLoS Pathog 2: 933-948.

Oliveira NM, Martinez-Garcia E, Xavier J, Durham WM, Kolter R, Kim W et al. (2015). Biofilm formation as a response to ecological competition. PLoS Biol 13: e1002191.

Papenfort K, Förstner KU, Cong J-P, Sharma CM, Bassler BL. (2015). Differential RNA-seq of Vibrio cholerae identifies the VqmR small RNA as a regulator of biofilm formation. Proc Natl Acad Sci USA 112: E766-E775.

Schluter J, Nadell CD, Bassler BL, Foster KR. (2015). Adhesion as a weapon in microbial competition. ISME J 9: 139-149.

Sengupta C, Mukherjee O, Chowdhury R. (2016). Adherence to intestinal cells promotes biofilm formation in Vibrio cholerae. J Infect Dis 214: 1571-1578.

Skorupski K, Taylor RK. (1996). Positive selection vectors for allelic exchange. Gene 169: 47-52.

Smith DR, Maestre-Reyna M, Lee G, Gerard H, Wang AH-J, Watnick PI. (2015). In situ proteolysis of the Vibrio cholerae matrix protein RbmA promotes biofilm recruitment. Proc Natl Acad Sci USA 112: 10491-10496.

Snell-Rood EC. (2013). An overview of the evolutionary causes and consequences of behavioural plasticity. Anim Behav 85: 1004-1011.

Stewart PS, Franklin MJ. (2008). Physiological heterogeneity in biofilms. Nat Rev Microbiol 6: 199-210.
Stewart PS. (2012). Mini-review: convection around biofilms. Biofouling 28: 187-198.

Stocker R, Seymour JR, Samandani A, Hunt DE, Polz MF. (2008). Rapic chemotactic response enables marine bacteria to exploit ephemeral microscale nutrient patches. Proc Natl Acad Sci USA 105: 4209-4214.

Stocker R, Seymour JR. (2012). Ecology and physics of bacterial chemotaxis in the ocean. Microbiol Mol Biol Rev 76: 792-812.

Teschler JK, Zamorano-Sanchez D, Utada AS, Warner CJA, Wong GCL, Linington RG et al. (2015). Living in the matrix: assembly and control of Vibrio cholerae biofilms. Nat Rev Microbiol 13: 255-268.

Utada AS, Bennett RR, Fong JCN, Gibiansky ML, Yildiz FH, Golestanian R et al. (2014). Vibrio cholerae use pili and flagella synergistically to effect motility switching and conditional surface attachment. Nat Commun 5: 4913.

Whiteley M, Bangera MG, Bumgarner RE, Parsek MR, Teitzel GM, Lory S et al. (2001). Gene expression in Pseudomonas aeruginosa biofilms. Nature 413: 860-864.

Wilson DS, Yoshimura J. (1994). On the coexistence of specialists and generalists. Am Nat 144: 692-707.

Xavier JB, Foster KR. (2007). Cooperation and conflict in microbial biofilms. Proc Natl Acad Sci USA 104: 876-881.

Yan J, Sharo AG, Stone HA, Wingreen NS, Bassler BL. (2016). Vibrio cholerae biofilm growth program and architecture revealed by single-cell live imaging. Proc Natl Acad Sci USA 113: E5337-E5343.

Yawata Y, Cordero OX, Menolascina F, Hehemann J-H, Polz MF, Stocker R. (2014). Competition-dispersal tradeoff ecologically differentiates recently speciated marine bacterioplankton populations. Proc Natl Acad Sci USA 111: 5622-5627.

Supplementary Information accompanies this paper on The ISME Journal website (http://www.nature.com/ismej) 